\title{
Evolving Role of Immunotherapy in Recurrent Metastatic Head and Neck Cancer
}

\author{
Xiuning Le, MD, PhD'; Renata Ferrarotto, $\mathrm{MD}^{1}$; Trisha Wise-Draper, $\mathrm{MD}, \mathrm{PhD}^{2}$; and Maura Gillison, $\mathrm{MD}, \mathrm{PhD}^{1}$
}

\section{ABSTRACT}

Immunotherapy has revolutionized cancer treatment in the past 2 decades, mostly with immune checkpoint blockade approaches. In squamous cell carcinoma of the head and neck (SCCHN), the initial efficacy of immunotherapy was observed in patients with recurrent or metastatic $(\mathrm{R} / \mathrm{M})$ disease who received other prior systemic treatment. As monotherapy, anti-PD-1 therapies induce responses in $13 \%$ to $18 \%$ of patients. More recently, immunotherapy in combination with cytotoxic chemotherapy demonstrated greater safety and efficacy as first-line systemic treatment compared with chemotherapy alone. In R/M SCCHN, the most important benefit of immunotherapy is the significantly improved overall survival, especially in patients with PD-L1-positive tumors. As of 2019, immunotherapy can be used as first-line or subsequent treatment of R/M SCCHN. Many ongoing trials are evaluating immunotherapy combinations or novel immunotherapy strategies, aiming to improve response rate and overall survival. As new targets are identified and new approaches are leveraged, the role of immunotherapy in R/M SCCHN continues to evolve.

J Natl Compr Canc Netw 2020;18(7):899-906 doi: $10.6004 /$ jnccn.2020.7590

\footnotetext{
${ }^{1}$ Department of Thoracic Head and Neck Medical Oncology, Division of Cancer Medicine, The University of Texas MD Anderson Cancer Center, Houston, Texas, and ${ }^{2}$ Department of Internal Medicine, Division of Hematology/Oncology, University of Cincinnati, Cincinnati, Ohio.
}

\section{Head and Neck Cancers}

Head and neck cancers account for $>700,000$ new cancer cases and 350,000 deaths annually worldwide. ${ }^{1}$ These cancers arise primarily from the mucosal surfaces lining the paranasal sinuses, nasal cavity, oral cavity, oropharynx, larynx, and hypopharynx, or from the major and minor salivary glands. Cancers may have a variety of histopathologic types, such as adenocarcinoma, adenoid cystic carcinoma, neuroendocrine carcinoma, or nonkeratinizing (eg, lymphoepithelioma) carcinoma. However, squamous cell carcinoma is the most common pathologic type. ${ }^{2}$

Squamous cell carcinoma of the head and neck (SCCHN) can be largely divided into 2 groups: those associated with carcinogens (eg, tobacco and alcohol) or with HPV infection. ${ }^{3}$ Most oral cavity, larynx, and hypopharynx cancers are tobacco- and alcohol-related. In contrast, most HPV-positive cancers arise from the oropharynx. ${ }^{3,4}$ HPV-positive cancers usually demonstrate high p16 expression by immunohistochemistry, which serves as a surrogate for HPV infection. ${ }^{5}$ Due to strong birth cohort effects, patients with HPV-positive cancer were on average younger than those with HPVnegative cancer, but the age difference is decreasing over time. ${ }^{6}$ Although oropharynx cancers in nonsmokers are attributable to HPV infection, most patients with HPVpositive oropharynx in the United States have a history of tobacco exposure, ${ }^{7,8}$ and response to therapy among this "intermediate-risk" group is less favorable compared with never-smokers. ${ }^{8}$ In both the United States and Europe, the incidence of HPV-associated SCCHN has been increasing, whereas the tobacco- and alcoholrelated SCCHN has been decreasing., ${ }^{4,9,10}$ In Asian countries, cases of tobacco- and alcohol-related SCCHN and Epstein-Barr virus-associated nasopharyngeal cancer remain dominant.

\section{Recurrent or Metastatic SCCHN}

Most patients with SCCHN have locoregionally limited disease at diagnosis, with only $10 \%$ of patients presenting with distant metastasis. ${ }^{11}$ Primary surgery followed by risk-adapted radiation with or without chemotherapy or with definitive concurrent chemoradiation are the 
principal treatment strategies for locoregionally advanced disease. Even with recent advances in surgery and radiation, however, a subset of patients will eventually experience disease progression. For example, locoregional or distant failure occurs in approximately $40 \%$ and $20 \%$, respectively, of patients with locoregionally advanced, HPV-negative SCCHN treated with cisplatin-based chemoradiotherapy. ${ }^{12}$ Recurrent disease is often complex, requiring multidisciplinary care to select the optimal combination and sequence of treatment. When disease recurrence is limited to local or regional failures, treatment with curative-intent surgical salvage may be possible. Patients who are not candidates for salvage surgery may be candidates for curative-intent radiation or reirradiation with or without chemotherapy, depending on the time since prior treatment and previous treatmentrelated toxicity. Palliative systemic therapy is indicated in patients diagnosed with recurrent or metastatic (R/M) SCCHN not amenable to the locally curative options mentioned earlier.

During 2008 through 2019, cetuximab and platinum5-FU, also known as the EXTREME regimen, was the firstline systemic therapy of choice for R/M SCCHN based on results of a phase III trial. ${ }^{13}$ The addition of cetuximab to the platinum doublet significantly improved overall survival (OS) and progression-free survival (PFS) and yielded an objective response rate (ORR) of $36 \%$. This regimen, however, is associated with an $82 \%$ rate of grade $3 / 4$ toxicities and a $20 \%$ treatment discontinuation rate. In clinical practice, a taxane is frequently substituted for 5-FU and administered with or without cetuximab. ${ }^{14,15}$ Until 2016, the second-line options after platinumcontaining regimen were limited to monotherapy, including a taxane, methotrexate, or cetuximab.

\section{Initial Success of Anti-PD-1 Therapy in R/M SCCHN}

Immunotherapy with immune checkpoint blockade was initially evaluated in clinical trials in patients with treatment-refractory melanoma (ClinicalTrials.gov identifier: NCT00289627, a single-arm ipilimumab trial). ${ }^{16}$ Subsequently, immune checkpoint blockade has demonstrated clinical benefit in a number of solid tumors. ${ }^{17}$ PD-1 is a "death receptor" that causes apoptosis in T cells to regulate the immune response and prevent autoimmunity. ${ }^{18,19}$ Many tumors express the PD-L1 ligand, which upon binding to the PD-1 receptor results in apoptosis of T cells and thus immune evasion. ${ }^{20}$ These discoveries led to the development of monoclonal antibodies to the PD-1 receptor, such as pembrolizumab and nivolumab, both of which were FDA approved for platinum-refractory R/M SCCHN in 2016.

The first anti-PD-1 therapeutic trial in R/M SCCHN was KEYNOTE-012 (NCT01848834), a phase I/II single-arm trial investigating the safety, tolerability, and antitumor activity of pembrolizumab (a humanized IgG4 antibody of PD-1) in a variety of solid tumors, including advanced head and neck cancer (cohorts B and B2). Patients were heavily pretreated with chemotherapy, because many had received 4 previous lines of systemic therapy. In cohort B $(n=60)$, all tumors had PD-L1 expression (immunohistochemistry [IHC] antibody clone 22C3) of at least $1 \%$, whereas tumors in cohort B2 $(n=132)$ were not required to have PD-L1 expression. In this trial, the ORR in all patients was $18 \%$, with $4 \%$ complete response, and median PFS was 2.1 months. ${ }^{21,22}$ ORR in HPV-positive tumors was $24 \%$ (95\% CI, $13 \%-40 \%$ ) versus $16 \%$ (95\% CI, $10 \%-23 \%$ ) in HPV-negative tumors (a nonsignificant difference, due to overlapping $95 \% \mathrm{CI}$ in the 2 groups). ${ }^{22}$

KEYNOTE-055 (NCT02255097) was designed to evaluate the ORR with pembrolizumab in patients with R/M SCCHN who were platinum- and cetuximabrefractory. Among 171 patients treated, the ORR was $16 \%(95 \%$ CI, $11 \%-23 \%)$ and PFS was 2.1 months. In this study, $82 \%$ of tumors were PD-L1-positive (based on combined positive score [CPS]), and the ORR was $18 \%$ versus $12 \%$ in PD-L1-negative tumors. ${ }^{23}$ Response rates were similar regardless of HPV status: $16 \%(95 \% \mathrm{CI}$, 6\%-32\%) in HPV-positive patients and $15 \%$ (95\% CI, $10 \%-23 \%$ ) in HPV-negative patients. ${ }^{23}$

\section{Anti-PD-1 Therapy Established as \\ Second-Line Treatment}

CheckMate 141 was a large randomized phase III study in patients with platinum-refractory R/M SCCHN designed to evaluate a principal outcome of OS with nivolumab (a fully human IgG4 anti-PD-1 antibody) versus singleagent chemotherapy of investigator's choice. Eligible patients included those with disease progression within 6 months of receipt of cisplatin in the primary treatment setting (eg, as induction, concurrent, or adjuvant therapy) or for R/M disease. ${ }^{24}$ Patients were randomized 2:1 to nivolumab versus standard monotherapy (eg, cetuximab, methotrexate, docetaxel). A total of 347 patients received at least one dose of assigned therapy (236 in the nivolumab group and 111 in the standard therapy group). The ORR in the nivolumab was $13.3 \%$ versus $5.8 \%$ in the standard-therapy group $(P<.01)$, and median OS was significantly longer (7.7 vs 5.1 months with standard therapy; HR, 0.68; $P<.01^{25}$ ). With long-term follow-up at 24 months, the estimated 24-month OS rate was nearly tripled with nivolumab compared with standard-of-care therapy (16.9\% vs 6.0\%). ${ }^{24}$ Median PFS in the 2 groups was similar. The magnitude of benefit of nivolumab over standard therapy appeared stronger at a median follow-up of 5.1 months for patients who were PDL1-positive ( $\geq 1 \%$ by IHC PD-L1 antibody clone $28-8$ ) or had p16-positive tumors, but was similar after a 
minimum 24-month follow-up. ${ }^{24-26}$ Of note, nivolumab improved OS in the subset of patients $(21.6 \%)$ treated in the first-line (hazard ratio [HR], 0.56; 95\% CI, 0.33-0.95), leading to a 1-year OS rate of $39.2 \%$ versus $15.4 \%$ with single-agent chemotherapy. ${ }^{26}$ The nivolumab arm also demonstrated less frequent treatment-related adverse events (trAEs) compared with standard-of-care therapy (all-grade trAEs: $58.9 \%$ vs $77.5 \%$; grade $3-4$ trAEs: $13.1 \%$ vs $35.1 \%) .{ }^{24}$

Similar to CheckMate 141, KEYNOTE-040 was a randomized phase III study comparing pembrolizumab versus standard-therapy (eg, cetuximab, methotrexate, or docetaxel) with a total of 595 patients enrolled. Response rates were $14.6 \%$ in the pembrolizumab group and $10.1 \%$ in the standard therapy group, and median OS was 8.4 months versus 6.9 months, respectively (HR, 0.80 ; 95\% CI, 0.65-0.98; $P=.016)$. Patients with at least $1 \%$ PDL1 (IHC antibody clone 22C3) in the tumor $(78 \%$ of all patients) benefited more from pembrolizumab (HR, 0.74; 95\% CI, 0.58-0.93; $P<.01) .{ }^{27}$

In summary, for patients with $\mathrm{R} / \mathrm{M}$ SCCHN who have received prior platinum-based therapies, anti-PD-1 monotherapy induces ORRs ranging from $13.3 \%$ to $18 \% .^{24,25}$ These results were superior to those for the standard second-line therapy options. Most importantly, both of these large randomized phase III studies showed that immunotherapy conferred a survival benefit. ${ }^{24-27}$ In the biomarker analysis, ${ }^{23,27}$ PD-L1 positivity was associated with improved benefits with anti-PD-1 therapy. However, patients with PD-L1 $0 \%$ tumors still may benefit from immunotherapy, although with a lower response rate. Based on these data, the FDA approved both pembrolizumab (August 2016) and nivolumab (November 2016) for treatment of patients with R/M SCCHN who experience disease progression on or after platinum-based therapy. It should be noted, however, that nivolumab is also approved for in the first-line setting if disease progresses within 6 months of platinum therapy in the primary treatment setting. ${ }^{26}$

\section{Combination of Chemoimmunotherapy in R/M SCCHN}

KEYNOTE-048 was designed to evaluate single-agent pembrolizumab or combination pembrolizumab + chemotherapy as first-line therapy for R/M SCCHN. ${ }^{28,29}$ In this large phase III study, 882 patients with newly diagnosed R/M SCCHN were randomized to 3 treatment groups: pembrolizumab alone versus pembrolizumab/ cisplatin/5-FU versus cetuximab/cisplatin/5-FU. Pembrolizumab or cetuximab maintenance was allowed. In this 3-arm study, pembrolizumab alone was noninferior in the total population when compared with cetuximab chemotherapy, and was associated with improved OS in
CPS $\geq 1$ and CPS $\geq 20$ populations. Pembrolizumab + chemotherapy also improved OS in the total population, as well as those with CPS $\geq 1$ and CPS $\geq 20$, compared with cetuximab + chemotherapy. Neither pembrolizumab alone nor pembrolizumab + chemotherapy was associated with improved PFS. ORRs in the 3 arms were $16.9 \%$ (pembrolizumab), $42 \%$ (pembrolizumab + chemotherapy), and $36.0 \%$ (cetuximab + chemotherapy) ${ }^{28-30}$ However, the PFS in the total population was 2.3 to 3.4 versus 4.9 to 5.8 versus 5.0 to 5.2 months, respectively. The ontreatment death rates were approximately $10 \%$ in the cetuximab + chemotherapy group and $11 \%$ in the pembrolizumab + chemotherapy group..$^{29,30}$

Based on the clear OS benefit demonstrated in the 2 arms treated with anti-PD-1 therapy, in June 2019 the FDA granted approval for pembrolizumab alone in patients with CPS $>1$ R/M SCCHN, and pembrolizumab + chemotherapy as first-line therapy in all patients with R/M SCCHN.

\section{PD-L1-Based Biomarkers in SCCHN}

As data have accumulated, use of immunotherapy based on tumor PD-L1 testing has evolved (Table 1). In the biomarker analysis of KEYNOTE-012, PD-L1 expression was scored using 2 methods: the tumor proportion score (TPS) and the CPS. TPS was defined as the percentage of tumor cells with membranous PD-L1 expression by IHC using antibody clone 22C3. In contrast, CPS is defined as the number of tumor and tumor-associated immune cells that express PDL1 divided by the number of viable tumors cells $\times 100$. Both scores range from $0 \%$ to $100 \% .{ }^{21}$ With a cutpoint of CPS $\geq 1$, response rates were higher in patients with positive versus negative (CPS $<1)$ tumors $(21 \%$ vs $6 \% ; P=.023$ ) but were not different based on the same TPS cutpoints. ${ }^{22}$ In subsequent studies, PD-L1 CPS $\geq 1$ was used as a biomarker cutpoint in pembrolizumab trials.

In KEYNOTE-055, 82\% of tumors had CPS $\geq 1$ (PDL1-positive). ORRs were $18 \%$ (95\% CI, 12\%-25\%) in PD-L1-positive patients versus $12 \%(95 \% \mathrm{CI}, 2 \%-30 \%)$ in PD-L1-negative patients, indicating similar benefit. Exploration of a different cutpoint of CPS $\geq 50$ observed in $29 \%$ of tumors demonstrated an ORR of $27 \%$ (95\% CI, $15 \%-42 \%)$. The confidence bounds overlapped with groups defined by CPS $<1$ and $\geq 1$. $^{23}$

In KEYNOTE-040, $79 \%$ of tumors in the pembrolizumabalone group had CPS $\geq 1$ and $26 \%$ had CPS $\geq 50$. Patients with CPS $\geq 1$ tumors had improved OS compared with those treated with standard-of-care chemotherapy (HR, 0.74; 95\% CI, 0.58-0.93; $P<.001$ ), and the benefit was stronger in the TPS $\geq 50$ group in the pembrolizumab alone group (HR, 0.53; 95\% CI, 0.35-0.81; $P<.001)$. In contrast, pembrolizumab did not benefit 


\begin{tabular}{|c|c|c|c|c|c|c|}
\hline Trials & $\begin{array}{l}\text { PD-L1 } \\
\text { Staining Cells }\end{array}$ & $\begin{array}{l}\text { Eligibility } \\
\text { PD-L1 Required }\end{array}$ & $\begin{array}{l}\text { Antibody } \\
\text { Used }\end{array}$ & $\begin{array}{l}\text { Population } \\
\text { Defined }\end{array}$ & $\begin{array}{l}\text { Response in } \\
\text { Total Population }\end{array}$ & $\begin{array}{l}\text { Response by PD-L1 } \\
\text { Subgroups }\end{array}$ \\
\hline $\begin{array}{l}\text { KEYNOTE-012, } \\
\text { cohort } \mathrm{B}^{21,22}\end{array}$ & Tumor cells (TPS) & Yes, $\geq 1 \%$ & $22 \mathrm{C} 3$ & NA & $18 \%$ & $\begin{array}{l}\text { ORR, } 21 \% \text { in } \mathrm{CPS} \geq 1 \text { vs } 6 \% \text { in } \\
\mathrm{CPS}<1\end{array}$ \\
\hline $\begin{array}{l}\text { KEYNOTE-012, } \\
\text { cohort B2 } 2^{21,22}\end{array}$ & Tumor cells (TPS) & No & & & & \\
\hline KEYNOTE- $055^{23}$ & CPS & No & $22 \mathrm{C} 3$ & $82 \%$ CPS $\geq 1$ & $16 \%$ & $\begin{array}{l}\text { ORR, } 18 \% \text { in } C P S \geq 1 \text { vs } 12 \% \text { in } \\
\text { CPS }<1 ; \\
\text { ORR, } 27 \% \text { in } C P S \geq 50 \text { group }\end{array}$ \\
\hline KEYNOTE-04027 & CPS and TPS & No & $22 \mathrm{C} 3$ & $\begin{array}{l}79 \% \text { CPS } \geq 1 \\
26 \% \text { TPS } \geq 50\end{array}$ & $\begin{array}{l}14.6 \% \text { with } \\
\text { immunotherapy }\end{array}$ & $\begin{array}{l}\mathrm{HR}, 0.74(95 \% \mathrm{Cl}, 0.58-0.93) \text { for } \\
\text { survival in } \mathrm{CPS} \geq 1 \text {; } \\
\mathrm{HR}, 0.53(95 \% \mathrm{Cl}, 0.35-0.81) \text { for } \\
\text { survival in TPS } \geq 50\end{array}$ \\
\hline CheckMate $141^{24-26}$ & Tumor cells & No & $28-8$ & $57 \%$ PD-L1 $\geq 1$ & $\begin{array}{l}13.3 \% \text { with } \\
\text { immunotherapy }\end{array}$ & $\begin{array}{l}\mathrm{HR}, 0.55(95 \% \mathrm{Cl}, 0.36-0.83) \text { for } \\
\text { survival in PD-L1 } \geq 1\end{array}$ \\
\hline KEYNOTE- $048^{28}$ & CPS & No & $22 \mathrm{C} 3$ & $\begin{array}{l}85 \% \text { CPS } \geq 1 \\
43 \% \text { CPS } \geq 20\end{array}$ & $\begin{array}{l}16.9 \% \text { with } \\
\text { immunotherapy }\end{array}$ & $\begin{array}{l}\text { 12-month survival CPS } \geq 20: \\
23 \% \text { (pembrolizumab) } \\
24 \% \text { (pembrolizumab }+ \\
\text { chemotherapy) } \\
11 \%-12 \% \text { (EXTREME) } \\
12-\text { month survival CPS } \geq 1: \\
20 \% \text { (pembrolizumab) } \\
19 \% \text { (pembrolizumab + } \\
\text { chemotherapy) } \\
11 \%-12 \% \text { (EXTREME) } \\
12-\text { onth survival total: } \\
17 \% \text { (pembrolizumab) } \\
17 \% \text { (pembrolizumab + } \\
\text { chemotherapy) } \\
12 \%-14 \% \text { (EXTREME) }\end{array}$ \\
\hline
\end{tabular}

Abbreviations: CPS, combined positive score; HR, hazard ratio; NA, not applicable; ORR, overall response rate; R/M, recurrent or metastatic; SCCHN, squamous cell carcinoma of the head and neck; TPS, tumor proportion score.

patients with CPS $<1$ tumors (HR, 1.28; 95\% CI, 0.80-2.07; $P=.84)^{27}$

The CheckMate 141 biomarker analysis used PD-L1 TPS with a different antibody (clone 28-8). However, in the Blueprint PD-L1 IHC Assay Comparison Poject for lung cancer, tumor and immune cell scoring by pathologists was comparable for clones $28-8$ and $22 \mathrm{C} 3 .^{31}$ In CheckMate 141, tumor TPS cutpoints of $\geq 1, \geq 5$, and $\geq 10$ were evaluated. Among 260 evaluable tumors, 57\% had PD-L1 TPS $\geq 1$, with an HR for death of 0.55 (95\% CI, 0.36-0.83). Analogous to KEYNOTE-040, the group with tumor PD-L1 TPS $<1$ did not experience significant benefit from immunotherapy. ${ }^{24}$

In KEYNOTE-048, further stratification of tumor PDL1 CPS was performed at cutpoints of $\geq 1$ and $\geq 20$, representing $85 \%$ and $43 \%$ of patients, respectively. As discussed in the previous session, pembrolizumab alone prolonged OS in the CPS $\geq 20$ and CPS $\geq 1$ groups. For patients with CPS $\geq 20$, the 12-month OS rate with pembrolizumab alone was $23 \% .^{28}$

In conclusion, biomarker analyses have established that PD-L1 is a reasonable biomarker for enrichment of survival benefit from anti-PD-1 immunotherapy in the first and subsequent line for R/M SCCHN (Table 1). Most patients have PD-L1-positive tumors
$(57 \%-82 \%) .^{23,24}$ Ongoing trials with additional antiPD-1/L1 agents, such as atezolizumab (NCT03818061), will provide additional analyses.

\section{Clinical Practice After the Addition of Immunotherapy}

When incorporating clinical trial evidence into general oncology practice, some practical aspects must be taken into consideration, such as the need for immediate symptom relief, short- versus long-term benefit from the therapy, toxicity, quality of life, logistical convenience of treatment administration, and financial burden. One major challenge to implementing the KEYNOTE-048 platinum + 5-FU/pembrolizumab combination is 5-FU-associated toxicity and the need for central venous access for continuous infusion.

At the annual ASCO 2019 meeting, results from the randomized phase III TPEx trial were reported..$^{15}$ This trial of 539 patients with newly diagnosed R/M SCCHN was designed to evaluate the noninferiority of cisplatin/ docetaxel/cetuximab for 4 cycles versus cetuximab/cisplatin/ 5-FU for 6 cycles (also known as the EXTREME regimen ${ }^{13}$ ). ${ }^{14}$ The cisplatin/docetaxel/cetuximab arm was shown to be noninferior to the EXTREME regimen with regard to OS, and neither PFS nor ORR showed any statistical 
difference. However, grade $\geq 4$ toxicity with taxane was significantly less compared with 5 -FU ( $34 \%$ vs $50 \%$; $P<.001)$. Due to the manageable toxicity profile and the convenience of same-day administration, docetaxel is viewed as a reasonable replacement for $5-\mathrm{FU}$, although the platinum/taxane/pembrolizumab combination has not been formally evaluated.

Although pembrolizumab + chemotherapy was FDA approved for first-line therapy for all patients with R/M SCCHN, pembrolizumab alone or cetuximab + chemotherapy may be preferred in some cases. In the KEYNOTE-048 trial, pembrolizumab + chemotherapy was associated with improved OS in the PD-L1 CPS $\geq 1$ population when compared with cetuximab + chemotherapy, but there was no subgroup analysis for patients whose tumors had $0 \%$ CPS ( $15 \%$ of patients). Furthermore, in the PD-L1-unselected population, pembrolizumab alone did not demonstrate a survival benefit compared with the EXTREME regimen or pembrolizumab + chemotherapy. ${ }^{28}$ Therefore, we recommend careful selection of therapy for patients with tumors of CPS $<1$ For patients with large disease burden and positive CPS $\geq 1$, who are otherwise healthy with excellent functional status to tolerate platinum doublet/pembrolizumab, the combination is the most appropriate first-line therapy.

\section{Novel Combinations and Strategies}

Despite the exciting progress with anti-PD-1 therapy with or without chemotherapy in patients with R/M SCCHN, response rates remain discouraging. When used as a single agent, anti-PD-1 therapies only achieve response in $<20 \%$ of patients regardless of the lines of therapy (ORRs of $18 \%$ in KEYNOTE- $012^{21} ; 14.6 \%$ in KEYNOTE-04027 $16 \%$ in KEYNOTE- $055^{23}$; $13.3 \%$ in CheckMate $141^{24}$; and $16.9 \%$ in KEYNOTE-048 ${ }^{28}$ ). Chemoimmunotherapy combinations used in the first-line setting have increased this response rate, but novel therapy combinations or novel immunotherapy strategies are desperately needed.

Many immunotherapy combinations have been tested in R/M SCCHN to evaluate whether adding a second or third immune checkpoint blockade can enhance anti-PD1/L1 efficacy. For example, CTLA-4 is another immune checkpoint protein that is expressed on $\mathrm{T}$ cells (mostly T-regulatory cells) to downregulate immune responses and mediates tumor immune escape. ${ }^{32-34}$ Furthermore, antiCTLA-4 in addition to anti-PD-1/L1 has been shown to improve response in patients with melanoma ${ }^{35,36}$ and possibly those with NSCLC. ${ }^{37,38}$ But anti-CTLA-4 plus anti-PD-Ll combinations reported to date have not shown additional benefit in R/M SCCHN. In the phase II randomized CONDOR study, the addition of tremelimumab (a human IgG2 antibody for CTLA-4) to durvalumab (a human IgG1 antibody for PD-L1) was safe and tolerable but did not add significant benefit regarding response or survival in patients whose tumors had low or zero PD-L1 expression $(<25 \%$ tumor cells expressing PD-L1 by SP263 assay). ${ }^{39}$ Similar results were reported from the phase III EAGLE trial, in which durvalumab was given with or without tremelimumab and compared with standard-ofcare second-line therapies in patients with R/M SCCHN. The primary endpoint was not met, because the addition of tremelimumab did not improve OS. Response rates were not different among all 3 groups. ${ }^{40}$ It was reported that IDO expression was associated with poor prognosis in SCCHN, and other cancer types, likely due to the inhibitory effect of IDO on T cells. ${ }^{41}$ Although inhibition of IDO in combination with pembrolizumab initially showed promising results in a phase I study (ECHO-202/ KEYNOTE-037), including an ORR of $34 \%(13 / 38)$ in patients with SCCHN, ${ }^{42}$ subsequent studies in melanoma (ECHO-301/KEYNOTE-252) failed to confirm the efficacy $^{43}$ and the SCCHN trial was halted. Additional immune checkpoints, for example, TIM-3 (NCT03652077 and NCT03307785), LAG3 (NCT04080804 and NCT03538028), and TIGIT (NCT04047862 and NCT02913313), are now being tested in early-phase clinical trials.

In addition to immune checkpoint inhibition, therapies targeting key cytokines and their pathways to induce immune modulation is another area of active immunotherapy research. The TGF- $\beta$ pathway represents a promising immune modulation target in HPVassociated diseases. ${ }^{44}$ Phase I trials (NCT02517398 and NCT02699515) evaluated M7824, a bifunctional fusion protein composed of 2 extracellular domains of TGF- $\beta$ RII (a TGF- $\beta$ "trap") fused with a human IgG1 monoclonal antibody against PD-L1, ${ }^{45}$ in $\mathrm{HPV}$-associated solid tumors. Response rates ranged between $28 \%$ to $37 \%$ in HPV-associated cases. ${ }^{46,47}$ Confirmative studies are ongoing. VEGF-A and VEGF receptor pathway inhibition has shown efficacy in combination with immune checkpoint blockade in other solid tumors. The combination of lenvatinib with pembrolizumab has demonstrated some initial promise in SCCHN. Lenvatinib is a multikinase inhibitor of vascular endothelial growth factor receptor (VEGFR) 1-3, fibroblast growth factor receptor (FGFR) 1-4, platelet-derived growth factor receptor (PDGFR) $\alpha$, RET, and KIT. A phase II trial in R/M SCCHN cancer reported a $36 \%$ ORR in 22 patients. ${ }^{48}$ Based on these data, an ongoing randomized placebo-controlled phase III trial (LEAP 010) is comparing this combination versus pembrolizumab alone. Additional combinations aiming to favorably modulate the tumor immune environment are in early-phase clinical trials, including combinations with antiSTAT3 (NCT03153982), PARP inhibitors, and cetuximab (NCT03082534 and NCT03691714).

Beyond immune checkpoint blockade and immune modulation, novel immune therapy strategies, such as vaccines, intratumor immunotherapy, and 
cellular therapy, have also been explored. Because the unique oncogenesis of HPV-associated SCCHN, targeting HPV16 (the most prevalent viral genotype) oncoproteins using a therapeutic vaccine approach has a solid biologic foundation and has achieved initial success. One example is the ISA101 vaccine, which is a synthetic long peptide HPV16 vaccine consisting of 13 overlapping peptides from the HPV16 oncoproteins E6 and E7. It has demonstrated an ORR of $36 \%(8 / 22)$ when combined with nivolumab in patients with oropharyngeal cancers. ${ }^{49,50} \mathrm{~A}$ randomized trial is ongoing to confirm the observed efficacy (NCT03669718). Other vaccines targeting either HPV oncoprotein or patient-specific neoantigens after whole-exon sequencing (GEN-009 trial; NCT03633110) are under active clinical investigation.

Intratumor injection of novel immunotherapies is especially appealing for SCCHN because of the technical feasibility of accessing the primary tumor or lymph nodes, especially for patients with locoregionally $\mathrm{R} / \mathrm{M}$ SCCHN. Intratumor administration of antitumor agents can be used with or without combinations of systemic immune checkpoint blockade or cytotoxic chemotherapies. With this strategy, an experienced head and neck surgical team, as well as an interventional radiology team, begins to play an increasingly important multidisciplinary role in palliative therapy for R/M SCCHN. Ongoing trials are evaluating agonist anti-CD40 (NCT03818542) and antiTLR (REVEAL trial; NCT03435640) and other immune therapy agents.

Last but not least, cellular therapy has been explored in solid tumors since its success in hematologic malignancies. ${ }^{51-53}$ For SCCHN, infusion of expanded autologous tumor-infiltrating T cells (NCT03991741) or natural killer cells (NCT02507154) is being evaluated. In HPV-associated SCCHN, initial efficacy with engineered T-cell therapies has been observed, ${ }^{54}$ and more trials are ongoing to understand how to leverage cellular therapy to treat SCCHN. ${ }^{55}$

\section{Conclusions}

Anti-PD-1 immune checkpoint blockade therapy has demonstrated efficacy in R/M SCCHN by inducing responses in $13 \%$ to $18 \%$ of patients and prolonging OS. PD-L1-based biomarkers have shown utility in guiding selection of treatment options, which is especially useful for first-line therapy. Current NCCN Guidelines for Head and Neck Cancers recommend immunotherapy with or without chemotherapy for patients with R/M SCCHN. In the first-line setting, platinum with 5-FU + pembrolizumab is a reasonable choice for all patients, and pembrolizumab is recommended as monotherapy for patients whose tumor have CPS $\geq 1$ or $\geq 20$. In the subsequent-line setting, nivolumab or pembrolizumab is recommended if disease progression occurs during or after platinum therapy. ${ }^{56}$

Much effort is now focusing on designing immune therapy combinations to further improve response and duration of response in patients. Some clinical trials have shown that certain combinations did not yield expected benefit (such as CTLA-4), but other combinations demonstrated promising preliminary efficacy (such as VEGF/ FGFR inhibitor or TGF- $\beta$ inhibitor). Novel immune therapy strategies, such as therapeutic vaccines or cellular therapy, might also yield great efficacy, especially for HPVassociated SCCHN. Moreover, numerous ongoing trials are evaluating the use of those agents in newly diagnosed SCCHN in earlier stages. We expect to continue learning how to best use immunotherapies to benefit patients through more trials and clinical experience.

Submitted January 22, 2020; accepted for publication May 12, 2020

Disclosures: Dr. Le has disclosed that she receives consulting fees from Eli Lilly, AstraZeneca, EMD Serono, and grant/research support from Eli Lilly and Boehringer Ingelheim. Dr. Ferrarotto has disclosed that she receives consulting fees from Regeneron-Sanofi, Ayala Pharma, Klus Pharma, Medscape, and Cellestia Biotech; has received grant/research support from AstraZeneca, Merck, Genentech, Pfizer. Dr. Wise-Draper has disclosed that she receives consulting fees from Shattuck Laboratories, and grant/research support from Bristol-Myers Squibb, Merck, and Tesaro/GlaxoSmithKline. Dr. Gillison has disclosed that she receives consulting fees from Merck, Bristol-Myers Squibb, Roche, Genocea, EMD Serono, Bayer, New Link Genetics, Aspyrian Therapeutics, TRM Oncology, Amgen, AstraZeneca, and Celgene, and grant/research support from Bristol-Myers Squibb and Merck.

Correspondence: Maura Gillison, MD, PhD, Department of Thoracic Head and Neck Medical Oncology, Division of Cancer Medicine, The University of Texas MD Anderson Cancer Center, 1515 Holcombe Boulevard, Houston, TX 77030. Email: mgillison@mdanderson.org

\section{References}

1. Bray F, Ferlay J, Soerjomataram I, et al. Global cancer statistics 2018 : GLOBOCAN estimates of incidence and mortality worldwide for 36 cancers in 185 countries. CA Cancer J Clin 2018;68:394-424.

2. Howlader N, Noone AM, Krapcho M, eds. SEER Cancer Statistics Review, 1975-2014, National Cancer Institute. Available at: https://seer.cancer. gov/csr/1975_2014/. Based on November 2016 SEER data submission. Posted April 2017

3. Gillison ML, Koch WM, Capone RB, et al. Evidence for a causal association between human papillomavirus and a subset of head and neck cancers. J Natl Cancer Inst 2000;92:709-720.
4. Gillison ML, Chaturvedi AK, Anderson WF, et al. Epidemiology of human papillomavirus-positive head and neck squamous cell carcinoma. J Clin Oncol 2015;33:3235-3242.

5. Shi W, Kato H, Perez-Ordonez B, et al. Comparative prognostic value of HPV16 E6 mRNA compared with in situ hybridization for human oropharyngeal squamous carcinoma. J Clin Oncol 2009;27:6213-6221.

6. Tota JE, Best AF, Zumsteg ZS, et al. Evolution of the oropharynx cancer epidemic in the United States: moderation of increasing incidence in younger individuals and shift in the burden to older individuals. J Clin Oncol 2019;37:1538-1546. 
7. Chaturvedi AK, D'Souza G, Gillison ML, et al. Burden of HPV-positive oropharynx cancers among ever and never smokers in the U.S. population. Oral Oncol 2016;60:61-67.

8. Ang KK, Harris J, Wheeler R, et al. Human papillomavirus and survival of patients with oropharyngeal cancer. N Engl J Med 2010;363:24-35.

9. Carlander AF, Grønhøj Larsen C, Jensen DH, et al. Continuing rise in oropharyngeal cancer in a high HPV prevalence area: a Danish population-based study from 2011 to 2014. Eur J Cancer 2017;70:75-82.

10. Louie KS, Mehanna H, Sasieni P. Trends in head and neck cancers in England from 1995 to 2011 and projections up to 2025. Oral Oncol 2015; 51:341-348.

11. Lydiatt WM, Patel SG, O'Sullian B, et al. Head and neck cancers-major changes in the American Joint Committee on Cancer Eighth Edition Cancer Staging Manual. CA Cancer J Clin 2017;67:122-137. doi:10.3322/ caac. 21389

12. Ang KK, Zhang $\mathrm{Q}$, Rosenthal DI, et al. Randomized phase III trial of concurrent accelerated radiation plus cisplatin with or without cetuximab for stage III to IV head and neck carcinoma: RTOG 0522. J Clin Oncol 2014;32:2940-2950.

13. Vermorken JB, Mesia R, Rivera F, et al. Platinum-based chemotherapy plus cetuximab in head and neck cancer. N Engl J Med 2008;359:1116-1127.

14. Guigay J, Keilholz U, Mesia R, et al. TPExtreme randomized trial: TPEx versus Extreme regimen in 1st line recurrent/metastatic head and neck squamous cell carcinoma [abstract]. J Clin Oncol 2015;33(Suppl):Abstract TPS6087.

15. Guigay J, Fayette J, Mesia R, et al. TPExtreme randomized trial: TPEx versus Extreme regimen in 1 st line recurrent/metastatic head and neck squamous cell carcinoma (R/M HNSCC) [abstract]. J Clin Oncol 2019; 37(Suppl):Abstract 6002.

16. Weber JS, O'Day S, Urba W, et al. Phase I/II study of ipilimumab for patients with metastatic melanoma. J Clin Oncol 2008:26:5950-5956.

17. Ribas A, Wolchok JD. Cancer immunotherapy using checkpoint blockade. Science 2018;359:1350-1355.

18. Zhu X, Lang J. Soluble PD-1 and PD-L1: predictive and prognostic significance in cancer. Oncotarget 2017;8:97671-97682.

19. Ishida Y, Agata Y, Shibahara K, et al. Induced expression of PD-1, a novel member of the immunoglobulin gene superfamily, upon programmed cell death. EMBO J 1992;11:3887-3895.

20. Dong H, Strome SE, Salomao DR, et al. Tumor-associated B7-H1 promotes T-cell apoptosis: a potential mechanism of immune evasion. Nat Med 2002;8:793-800

21. Seiwert TY, Burtness B, Mehra R, et al. Safety and clinical activity of pembrolizumab for treatment of recurrent or metastatic squamous cell carcinoma of the head and neck (KEYNOTE-012): an open-label, multicentre, phase $1 \mathrm{~b}$ trial. Lancet Oncol 2016;17:956-965.

22. Mehra R, Seiwert TY, Gupta S, et al. Efficacy and safety of pembrolizumab in recurrent/metastatic head and neck squamous cell carcinoma: pooled analyses after long-term follow-up in KEYNOTE-012. Br J Cancer 2018; 119:153-159.

23. Bauml J, Seiwert TY, Pfister DG, et al. Pembrolizumab for platinum- and cetuximab-refractory head and neck cancer: results from a single-arm, phase II study. J Clin Oncol 2017;35:1542-1549.

24. Ferris RL, Blumenschein G Jr, Fayette J, et al. Nivolumab for recurrent squamous-cell carcinoma of the head and neck. N Engl J Med 2016;375: 1856-1867.

25. Ferris RL, Blumenschein G Jr, Fayette J, et al. Nivolumab vs investigator's choice in recurrent or metastatic squamous cell carcinoma of the head and neck: 2-year long-term survival update of CheckMate 141 with analyses by tumor PD-L1 expression. Oral Oncol 2018;81:45-51.

26. Gillison ML, Blumenschein G Jr, Fayette J, et al. CheckMate 141: 1-year update and subgroup analysis of nivolumab as first-line therapy in patients with recurrent/metastatic head and neck cancer. Oncologist 2018;23: 1079-1082.

27. Cohen EEW, Soulières D, Le Tourneau C et al. Pembrolizumab versus methotrexate, docetaxel, or cetuximab for recurrent or metastatic headand-neck squamous cell carcinoma (KEYNOTE-040): a randomised, openlabel, phase 3 study. Lancet 2019;393:156-167.

28. Burtness B, Harrington KJ, Greil R, et al. Pembrolizumab alone or with chemotherapy versus cetuximab with chemotherapy for recurrent or metastatic squamous cell carcinoma of the head and neck (KEYNOTE048): a randomised, open-label, phase 3 study. Lancet 2019;394 1915-1928.

29. Burtness B, Harrington KJ, Greil R, et al. KEYNOTE-048: phase 3 study of first-line pembrolizumab (P) for recurrent/metastatic head and neck squamous cell carcinoma (R/M HNSCC). Ann Oncol 2018;29(Suppl 8): VIII729.

30. Rischin D, Harrington KJ, Greil R, et al. Protocol-specified final analysis of the phase 3 KEYNOTE-048 trial of pembrolizumab (pembro) as first-line therapy for recurrent/metastatic head and neck squamous cell carcinoma (R/M HNSCC) [abstract]. J Clin Oncol 2019;37(Suppl):Abstract 6000.

31. Hirsch FR, McElhinny A, Stanforth D, et al. PD-L1 immunohistochemistry assays for lung cancer: results from phase 1 of the Blueprint PD-L1 IHC Assay Comparison Project. J Thorac Oncol 2017;12:208-222.

32. Baitsch $L$, Baumgaertner $P$, Devêvre $E$, et al. Exhaustion of tumor-specific $\mathrm{CD}^{+} \mathrm{T}$ cells in metastases from melanoma patients. J Clin Invest 2011 121:2350-2360.

33. Tivol EA, Borriello F, Schweitzer AN, et al. Loss of CTLA-4 leads to massive lymphoproliferation and fatal multiorgan tissue destruction, revealing a critical negative regulatory role of CTLA-4. Immunity 1995;3:541-547.

34. Waterhouse P, Penninger JM, Timms E, et al. Lymphoproliferative disorders with early lethality in mice deficient in CTLA-4. Science 1995;270: 985-988.

35. Wolchok JD, Chiarion-Sileni V, Gonzalez R, et al. Overall survival with combined nivolumab and ipilimumab in advanced melanoma. N Engl J Med 2017;377:1345-1356.

36. Wolchok JD, Rollin L, Larkin J. Nivolumab and ipilimumab in advanced melanoma. N Engl J Med 2017;377:2503-2504.

37. Hellmann MD, Ciuleanu TE, Pluzanski A, et al. Nivolumab plus ipilimumab in lung cancer with a high tumor mutational burden. N Engl J Med 2018 378:2093-2104.

38. Hellmann MD, Paz-Ares L, Bernabe Caro R, et al. Nivolumab plus ipilimumab in advanced non-small-cell lung cancer. N Engl J Med 2019;381: 2020-2031.

39. Siu LL, Even C, Mesía R, et al. Safety and efficacy of durvalumab with or without tremelimumab in patients with PD-L1-low/negative recurrent or metastatic HNSCC: the phase 2 CONDOR randomized clinical trial. JAMA Oncol 2019;5:195-203.

40. Licitra LF, Haddad RI, Even C, et al. EAGLE: a phase 3, randomized, openlabel study of durvalumab (D) with or without tremelimumab $(T)$ in patients (pts) with recurrent or metastatic head and neck squamous cell carcinoma (R/M HNSCC) [abstract]. J Clin Oncol 2019;37(Suppl):Abstract 6012.

41. Komiya T, Huang $\mathrm{CH}$. Updates in the clinical development of epacadostat and other indoleamine 2,3-dioxygenase 1 inhibitors (IDO1) for human cancers. Front Oncol 2018;8:423.

42. Mitchell TC, Hamid O, Smith DC, et al. Epacadostat plus pembrolizumab in patients with advanced solid tumors: phase I results from a multicenter open-label phase I/II trial (ECHO-202/KEYNOTE-037). J Clin Oncol 2018 36:3223-3230.

43. Long GV, Dummer R, Hamid O, et al. Epacadostat plus pembrolizumab versus placebo plus pembrolizumab in patients with unresectable or metastatic melanoma (ECHO-301/KEYNOTE-252): a phase 3, randomised, double-blind study. Lancet Oncol 2019;20:1083-1097.

44. Sporn MB, Roberts AB. TGF-beta: problems and prospects. Cell Regul 1990;1:875-882

45. Lan $Y$, Zhang $D, X u C$, et al. Enhanced preclinical antitumor activity of M7824, a bifunctional fusion protein simultaneously targeting PD-L1 and TGF- $\beta$. Sci Transl Med 2018;10:eaan5488.

46 Strauss J, Gatti-Mays ME, Tedman J, et al. Safety and activity of M7824, a bifunctional fusion protein targeting PD-L1 and TGF- $\beta$, in patients with HPV associated cancers [abstract]. J Clin Oncol 2018;36(Suppl):Abstract 3007.

47. Strauss J, Gatti-Mays ME, Cho B, et al. Phase I evaluation of M7824, a bifunctional fusion protein targeting TGF- $\beta$ and PD-L1, in patients with human papillomavirus (HPV)-associated malignancies [abstract]. Cancer Res 2019;79(13 Suppl):Abstract CT075.

48. Taylor MH, Rasco DW, Brose MS, et al. A phase $1 \mathrm{~b} / 2$ trial of lenvatinib plus pembrolizumab in patients with squamous cell carcinoma of the head and neck [abstract]. J Clin Oncol 2018;36(Suppl):Abstract 6016.

49. Glisson B, Massarelli E, William WN, et al. Nivolumab and ISA 101 HPV vaccine in incurable HPV-16+ cancer [abstract]. Ann Oncol 2017 28(Suppl):Abstract 2757.

50. Massarelli E, William W, Johnson F, et al. Combining immune checkpoint blockade and tumor-specific vaccine for patients with incurable human papillomavirus 16-related cancer: a phase 2 clinical trial. JAMA Oncol 2019;5:67-73.

51. Park JH, Rivière I, Gonen M, et al. Long-term follow-up of CD19 CAR therapy in acute lymphoblastic leukemia. N Engl J Med 2018;378: 449-459. 
52. Maude SL, Laetsch TW, Buechner J, et al. Tisagenlecleucel in children and young adults with B-cell lymphoblastic leukemia. N Engl J Med 2018;378: 439-448.

53. June $\mathrm{CH}$, Sadelain M. Chimeric antigen receptor therapy. N Engl J Med 2018;379:64-73.

54. Nagarsheth N, Norberg S, Doran SL, et al. Regression of epithelial cancers in humans following T-cell receptor gene therapy targeting human papillomavirus-16 E7 [abstract]. J Clin Oncol 2018;36(Suppl):Abstract 3043.
55. Blumenschein GR, Bourgogne A, Reinhardt C, et al. Phase I trial evaluating genetically modified autologous T cells (ACTengine IMA201) expressing a T-cell receptor recognizing a cancer/germline antigen in patients with squamous NSCLC or HNSCC [abstract]. J Clin Oncol 2018; 36(Suppl):Abstract TPS78.

56. Pfister DG, Spencer S, Adelstein D, et al. NCCN Clinical Practice Guidelines in Oncology: Head and Neck Cancers. Version 1.2020. Accessed May 20, 2020. To view the most recent version, visit NCCN.org

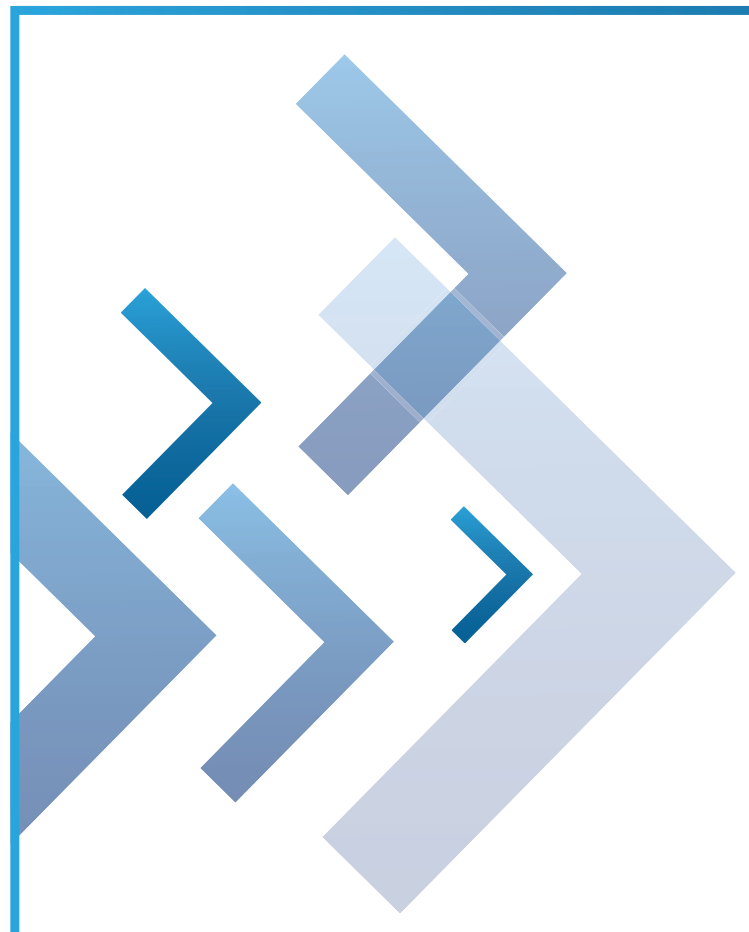

\section{NCCN \\ NATIONAL COMPREHENSIVE CANCER NETWORK

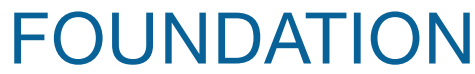 \\ Guiding Treatment. Changing Lives.}

You are invited to join the virtual

Friends of NCCN Foundation Supporter Forum

Two chances to attend!

Friday, July 24, 2020 | 11 AM - 12:30 PM EDT

Thursday, July 30, 2020 | 1 - 2:30 PM EDT

Learn how the NCCN Guidelines for Patients ${ }^{\circledR}$ are adapted and updated from the NCCN Clinical Practice Guidelines in Oncology (NCCN Guidelines ${ }^{\circledR}$ ).

\section{Register for free: NCCN.org/events}

